\title{
Uracil DNA glycosylase 2 negatively regulates HIV-I LTR transcription
}

\author{
David Fenard*1,2,3, Laurent Houzet 1,2,3, Audrey Tupin ${ }^{2,3}$, Sonia Brun ${ }^{2,3}$, \\ Marylène Mougel 2,3 , Christian Devaux ${ }^{2,3}$, Nathalie Chazal ${ }^{2,3}$ and \\ Laurence Briant ${ }^{2,3}$
}

Address: ${ }^{1}$ Université Montpellier 1, Centre d'études d'agents Pathogènes et Biotechnologies pour la Santé (CPBS), France, ${ }^{2} \mathrm{CNRS}, \mathrm{UMR} 5236$, CPBS, F-34965 Montpellier, France and ${ }^{3}$ Université Montpellier 2, CPBS, F-34095 Montpellier, France

* Corresponding author

from Frontiers of Retrovirology: Complex retroviruses, retroelements and their hosts

Montpellier, France. 21-23 September 2009

Published: 24 September 2009

Retrovirology 2009, 6(Suppl 2):P29 doi:10.1186/1742-4690-6-S2-P29

This abstract is available from: http://www.retrovirology.com/content/6/S2/P29

(c) 2009 Fenard et al; licensee BioMed Central Ltd.

Numerous cellular factors belonging to the DNA repair machineries, including RAD18, RAD52, XPB and XPD, have been described to counteract human immunodeficiency virus type 1 (HIV-1) replication. Recently, Uracil DNA glycosylase 2 (UNG2), a major determinant of the uracil base excision repair pathway, was shown to undergo rapid proteasome-dependent degradation following HIV-1 infection. However, the specific role of intracellular UNG2 depletion during the course of HIV-1 infection is not clearly understood. Our study shows for the first time that overexpression of UNG2 inhibits HIV-1 replication. We demonstrate that this viral inhibition is correlated with a marked decrease in transcription efficiency as shown by monitoring HIV-1 LTR promoter activity and quantification of HIV-1 RNA levels. Interestingly, UNG2 inhibits LTR activity when stimulated by Tat transactivator or TNF $\alpha$, while barely affected using Phorbol ester activation. Mutational analysis of UNG2 indicates that antiviral activity may require the integrity of the UNG2 catalytic domain and Vpr-interacting domain. Altogether, our data indicate that UNG2 is likely to represent a new host defense factor specifically counteracted by HIV-1 Vpr. The molecular mechanisms involved in the UNG2 antiviral activity still remain elusive but may rely on the sequestration of specific cellular factor(s) critical for viral transcription. 